J. Appl. Numer. Optim. 3 (2021), No. 3, pp. 479-487

Available online at http://jano.biemdas.com

https://doi.org/10.23952/jano.3.2021.3.03

\title{
TWO CONVERGENCE RESULTS FOR A GRADIENT-TYPE METHOD IN BANACH SPACES
}

\author{
ALEXANDER J. ZASLAVSKI
}

Department of Mathematics, The Technion - Israel Institute of Technology, Haifa, Israel

\begin{abstract}
Given a convex objective function on a Banach space which is Lipschitz on bounded sets and satisfies a coercivity growth condition, we prove two convergence results for a gradient-type method, generated by a regular vector field, under the presence of computational errors. We show that if the computational errors are small enough, then the values of the objective function become close to its infimum.
\end{abstract}

Keywords. Banach space; Complete metric space; Convex function; Descent method; gradient-type method.

\section{INTRODUCTION}

Given a Lipschitz convex and coercive objective function on a Banach space, we consider a complete metric space of vector fields, which are self-mappings of the Banach space, with the topology of uniform convergence on bounded subsets. With each such vector field, we associate a certain iterative process. The class of regular vector fields was introduced in $[1,2]$, where it was shown, using the generic approach and the porosity notion, that a typical vector field is regular and that for a regular vector field, the values of the objective function at the points generated by some iterative process tend to its infimum. Taking into account computational errors, we study in the present paper the behavior of the values of the objective function for a gradient-type method, generated by a regular vector field. We show that if the computational errors are small enough, then the values of the objective functions become close to its infimum.

Assume that $(X,\|\cdot\|)$ is a Banach space with norm $\|\cdot\|,\left(X^{*},\|\cdot\|_{*}\right)$ is its dual space with the norm $\|\cdot\|_{*}$, and $f: X \rightarrow R^{1}$ is a convex continuous function, which is bounded from below. Recall that, for each pair of sets $A, B \subset X^{*}$,

$$
H(A, B)=\max \left\{\sup _{x \in A} \inf _{y \in B}\|x-y\|_{*}, \sup _{y \in B} \inf _{x \in A}\|x-y\|_{*}\right\}
$$

is the Hausdorff distance between $A$ and $B$.

For each point $x \in X$, let

$$
\partial f(x)=\left\{l \in X^{*}: f(y)-f(x) \geq l(y-x) \text { for all } y \in X\right\}
$$

E-mail address: ajzasl@technion.ac.il.

Received May 12, 2021; Accepted September 30, 2021.

(C)2021 Journal of Applied and Numerical Optimization 
be the subdifferential of $f$ at $x$ [3]. It is well known that the set $\partial f(x)$ is a nonempty and bounded subset of $\left(X^{*},\|\cdot\|_{*}\right)$.

Set $\inf (f):=\inf \{f(x): x \in X\}$. Denote by $\mathscr{A}$ the set of all mappings $V: X \rightarrow X$ such that $V$ is bounded on every bounded subset of $X$ (that is, for each $K_{0}>0$, there is $K_{1}>0$ such that $\|V(x)\| \leq K_{1}$ if $\left.\|x\| \leq K_{0}\right)$ and, for each $x \in X$ and each $l \in \partial f(x), l(V(x)) \leq 0$. We denote by $\mathscr{A}_{c}$ the set of all continuous $V \in \mathscr{A}$, by $\mathscr{A}_{u}$ the set of all $V \in \mathscr{A}$, which are uniformly continuous on each bounded subset of $X$, and by $\mathscr{A}_{a u}$ the set of all $V \in \mathscr{A}$, which are uniformly continuous on the subsets

$$
\{x \in X:\|x\| \leq n \text { and } f(x) \geq \inf (f)+1 / n\}
$$

for each integer $n \geq 1$. Finally, let $\mathscr{A}_{a u c}=\mathscr{A}_{a u} \cap \mathscr{A}_{c}$.

Next, we endow the set $\mathscr{A}$ with a metric $\rho$. For each $V_{1}, V_{2} \in \mathscr{A}$ and each integer $i \geq 1$, we first set

and then define

$$
\rho_{i}\left(V_{1}, V_{2}\right):=\sup \left\{\left\|V_{1}(x)-V_{2}(x)\right\|: x \in X \text { and }\|x\| \leq i\right\}
$$

$$
\rho\left(V_{1}, V_{2}\right):=\sum_{i=1}^{\infty} 2^{-i}\left[\rho_{i}\left(V_{1}, V_{2}\right)\left(1+\rho_{i}\left(V_{1}, V_{2}\right)\right)^{-1}\right]
$$

Clearly $(\mathscr{A}, \rho)$ is a complete metric space. It is also not difficult to see that the collection of the sets

$$
E(N, \varepsilon)=\left\{\left(V_{1}, V_{2}\right) \in \mathscr{A} \times \mathscr{A}:\left\|V_{1}(x)-V_{2}(x)\right\| \leq \varepsilon, x \in X,\|x\| \leq N\right\},
$$

where $N, \varepsilon>0$, is a basis for the uniformity generated by the metric $\rho$. Evidently $\mathscr{A}_{c}, \mathscr{A}_{u}, \mathscr{A}_{a u}$, and $\mathscr{A}_{\text {auc }}$ are closed subsets of the metric space $(\mathscr{A}, \rho)$. In the sequel, we assign to all these spaces the same metric $\rho$.

In order to compute $\inf (f)$, we associate with each vector field $W \in \mathscr{A}$ a gradient-like iterative process.

The study of minimization methods for convex functions is a central topic in optimization theory; see, for example, $[4,5,6,7,8,9,10,11,12,13,14,15]$ and the references mentioned therein. Note, in particular, that the counterexample studied in Section 2.2 of Chapter VIII of [16] shows that, even for two-dimensional problems, the simplest choice for a descent direction, namely the normalized steepest descent direction,

$$
V(x)=\operatorname{argmin}\left\{\max _{l \in \partial f(x)}\langle l, d\rangle:\|d\|=1\right\},
$$

may produce sequences the functional values of which fail to converge to the infimum of $f$.

In infinite dimensional settings, the problem is even more difficult and less understood. Moreover, positive results usually require special assumptions on the space and the functions. However, in [1] (under certain assumptions on function $f$ ), for an arbitrary Banach space $X$, it was established the existence of a set $\mathscr{F}$, which is a countable intersection of open everywhere dense subsets of $\mathscr{A}$ such that, for any $V \in \mathscr{F}$, the values of $f$ tend to its infimum for some iterative process associated with $V$.

In [2], it was introduced the class of regular vector fields $V \in \mathscr{A}$ and it was shown (under the two mild assumptions $\mathrm{A}(\mathrm{i})$ and $\mathrm{A}$ (ii) on $f$ stated below) that the complement of the set of regular vector fields is not only of the first category, but also $\sigma$-porous in each of the spaces $\mathscr{A}$, $\mathscr{A}_{c}, \mathscr{A}_{u}, \mathscr{A}_{a u}$, and $\mathscr{A}_{a u c}$. It was shown in [2] that, for any regular vector field $V \in \mathscr{A}_{a u}$, the values of $f$ tend to its infimum for some iterative process associated with $V$ if, in addition to A(i) and A(ii), $f$ also satisfies assumption A(iii). Note that the results of [2] are also presented in [17, 
Chapter 8], which contains many other generic and porosity results. For more applications of the generic approach and the porosity notion in optimization theory, we refer to [18].

These results are established in any Banach space and for those convex functions satisfying the following two assumptions, which are also used in this paper.

A(i) There exists a norm-bounded set $X_{0} \subset X$ such that

$$
\inf (f)=\inf \{f(x): x \in X\}=\inf \left\{f(x): x \in X_{0}\right\}
$$

A(ii) for each $r>0$, the function $f$ is Lipschitz on the ball $\{x \in X:\|x\| \leq r\}$.

We may assume that the set $X_{0}$ in A(i) is closed and convex. It is clear that assumption A(i) holds if $\lim _{\|x\| \rightarrow \infty} f(x)=\infty$. We say that a mapping $V \in \mathscr{A}$ is regular if, for any natural number $n$, there exists a positive number $\delta(n)$ such that, for each point $x \in X$ satisfying

$$
\|x\| \leq n \text { and } f(x) \geq \inf (f)+1 / n,
$$

and each $l \in \partial f(x), l(V(x)) \leq-\delta(n)$. In this connection, see also [19].

In the sequel we also make use of the following assumption:

A(iii) For each integer $n \geq 1$, there exists $\delta>0$ such that, for each $x_{1}, x_{2} \in X$ satisfying

$$
\left\|x_{1}\right\|,\left\|x_{2}\right\| \leq n, f\left(x_{i}\right) \geq \inf (f)+1 / n, i=1,2, \text { and }\left\|x_{1}-x_{2}\right\| \leq \delta,
$$

the following inequality holds:

$$
H\left(\partial f\left(x_{1}\right), \partial f\left(x_{2}\right)\right) \leq 1 / n \text {. }
$$

This assumption is certainly satisfied if $f$ is differentiable and its derivative is uniformly continuous on those bounded subsets of $X$ over which the infimum of $f$ is larger than $\inf (f)$.

Note that a regular vector field was constructed in [17, Proposition 8.5]. In [19], it was obtained necessary and sufficient conditions for the regularity of descent vector-fields associated with convex and Lipschitz functions defined on general Banach spaces.

\section{THE FiRST RESULT}

For each $x \in X$ and each $r>0$, set

$$
B(x, r)=\{y \in X:\|x-y\| \leq r\} .
$$

Theorem 2.1. Assume that $f(x) \rightarrow \infty$ as $\|x\| \rightarrow \infty$, a vector field $V \in \mathscr{A}$ is regular, assumption A(ii) is valid and at least one of the following conditions holds: $1 . V \in \mathscr{A}_{\text {au }} ; 2$. A(iii) is valid. Let $K, \varepsilon>0$ be given. Then there exists $\beta_{0} \in(0,1]$ such that for each $\beta_{1} \in\left(0, \beta_{0}\right]$ there exist $\delta>0$ and a natural number $N_{0}$ such that, for each integer $n \geq N_{0}$, each sequence $\left\{a_{i}\right\}_{i=0}^{n-1} \subset\left[\beta_{1}, \beta_{0}\right]$, and each sequence $\left\{x_{i}\right\}_{i=0}^{n} \subset X$, which satisfies $\left\|x_{0}\right\| \leq K$ and $\left\|x_{i+1}-x_{i}-a_{i} V\left(x_{i}\right)\right\| \leq \delta$ for each $i=0, \ldots, n-1 f\left(x_{i}\right) \leq \inf (f)+\varepsilon$ holds for all integers $i \in\left[N_{0}, n\right]$.

\section{An Auxiliary Result}

In the proof of Theorem 2.1, we use the following lemma, which was proved in [20].

Lemma 3.1. Assume that $W \in \mathscr{A}$ is regular, $A(i), A(i i)$ are valid and that at least one of the following conditions holds: $1 . W \in \mathscr{A}_{\text {au }} ; 2$. A(iii) is valid. Let $\bar{K}$ and $\bar{\varepsilon}$ be positive. Then there exist positive numbers $\bar{\alpha}, \gamma$ and $\delta$ such that, for each point $x \in X$ satisfying $\|x\| \leq \bar{K}, f(x) \geq$ $\inf (f)+\bar{\varepsilon}$, each number $\beta \in(0, \bar{\alpha}]$, and each point $y \in B(W(x), \delta)$, we have $f(x)-f(x+\beta y) \geq$ $\beta \gamma$. 


\section{Proof of Theorem 2.1}

We may assume without loss of generality that $\varepsilon<1, K>2$ and that

$$
\{x \in X: f(x) \leq \inf (f)+4\} \subset B(0, K-2) .
$$

Let

$$
K_{0}>\sup \{f(x): x \in B(0, K+1)\}+\sup \{\|V(x)\|: x \in B(0, K+1)\} .
$$

Set

$$
E_{0}:=\left\{x \in X: f(x) \leq K_{0}+1\right\} .
$$

Clearly, the set $E_{0}$ is bounded and closed. Choose

$$
K_{1}>\max \left\{\sup \left\{\|x\|: x \in E_{0}\right\}+1+K, \sup \left\{\|V(x)\|: x \in E_{0}\right\}+1\right\} .
$$

There exists $L_{0} \geq 1$ such that

$$
|| f\left(z_{1}\right)-f\left(z_{2}\right) \mid \leq L_{0}\left\|z_{1}-z_{2}\right\| \text { for all } z_{1}, z_{2} \in B(0, K+2) .
$$

Lemma 3.1 implies that there exist positive numbers $\bar{\alpha}, \delta_{0} \in(0,1)$ and $\gamma>0$ such that the following property holds:

(a) for each point $x \in X$ satisfying

$$
\|x\| \leq K_{1}, f(x) \geq \inf (f)+\varepsilon / 4,
$$

each number $\alpha \in(0, \bar{\alpha}]$ and each point $y \in B\left(V(x), \delta_{0}\right)$,

$$
f(x)-f(x+\alpha y) \geq \alpha \gamma
$$

Fix a positive number

$$
\beta_{0} \leq \min \left\{\bar{\alpha},\left(L_{0}\left(K_{0}+1\right)\right)^{-1} \varepsilon / 4\right\} .
$$

Let $\beta_{1} \in\left(0, \beta_{0}\right]$. Fix a natural number

$$
N_{0}>\gamma^{-1}\left(K_{0}-\inf (f)\right) \beta_{1}^{-1}
$$

and

$$
\delta \in\left(0, \beta_{1} \delta_{0}\right]
$$

Assume that $n \geq N_{0}$ is an integer, $\left\{x_{i}\right\}_{i=0}^{n} \subset X$ satisfies

$$
\begin{gathered}
\left\|x_{0}\right\| \leq K, \\
\left\{a_{i}\right\}_{i=0}^{n-1} \subset\left[\beta_{1}, \beta_{0}\right],
\end{gathered}
$$

and

$$
\left\|x_{i+1}-x_{i}-a_{i} V\left(x_{i}\right)\right\| \leq \delta
$$

for all $i=0, \ldots, n-1$. In order to complete the proof, it is sufficient to show that $f\left(x_{i}\right) \leq$ $\inf (f)+\varepsilon$ for all integers $i \in\left[N_{0}, n\right]$.

First, we show that there exists an integer $j \in\left[0, N_{0}\right]$ such that $f\left(x_{j}\right) \leq \inf (f)+\varepsilon / 4$. Assume the contrary. Then

$$
f\left(x_{i}\right)>\inf (f)+\varepsilon / 4, i=0, \ldots, N_{0} .
$$

Assume that

$$
i \in\left\{0, \ldots, N_{0}\right\},\left\|x_{i}\right\| \leq K_{1} .
$$


Set $y_{i}=a_{i}^{-1}\left(x_{i+1}-x_{i}\right)$. It follows from (4.8), (4.10), and (4.11) that

$$
\begin{aligned}
\left\|y_{i}-V\left(x_{i}\right)\right\|=\left\|a_{i}^{-1}\left(x_{i+1}-x_{i}\right)-V\left(x_{i}\right)\right\| & \leq a_{i}^{-1}\left\|\left(x_{i+1}-x_{i}\right)-a_{i} V\left(x_{i}\right)\right\| \\
& \leq a_{i}^{-1} \delta \leq \beta_{1}^{-1} \delta \\
& \leq \delta_{0}
\end{aligned}
$$

and $x_{i+1}=x_{i}+a_{i} y_{i}$. It follows from property (a), (4.6), (4.10), (4.12), and (4.13) that

$$
f\left(x_{i}\right)-f\left(x_{i+1}\right) \geq a_{i} \gamma \geq \beta_{1} \gamma .
$$

Thus we have shown that the following property holds:

(b) if $i \in\left\{0, \ldots, N_{0}\right\},\left\|x_{i}\right\| \leq K_{1}$, then $f\left(x_{i}\right)-f\left(x_{i+1}\right) \geq \beta_{1} \gamma$. We show that $\left\|x_{i}\right\| \leq K_{1}, i=$ $0, \ldots, N_{0}$. Assume the contrary. Then there exists an integer $k \in\left\{0, \ldots, N_{0}\right]$ such that

$$
\left\|x_{k}\right\|>K_{1}
$$

In view of (4.4), (4.9), and (4.14), we have $k>0$. We may assume without loss of generality that

$$
\left\|x_{i}\right\| \leq K_{1}, i=0, \ldots, k-1 .
$$

Property (b), and (4.15) imply that, for each $i \in\{0, \ldots, k-1\}, f\left(x_{i+1}\right) \leq f\left(x_{i}\right)$. From (4.2) and (4.9), we obtain that $f\left(x_{k}\right) \leq f\left(x_{0}\right) \leq K_{0}$. Combining (4.3) and (4.4), we conclude that $\left\|x_{k}\right\| \leq K_{1}$. This contradicts (4.14). The contradiction we have reached proves that

$$
\left\|x_{i}\right\| \leq K_{1}, i=0, \ldots, N_{0} .
$$

Property (b) and (4.16) imply that, for all $i=0, \ldots, N_{0}-1$,

$$
f\left(x_{i}\right)-f\left(x_{i+1}\right) \geq \beta_{1} \gamma
$$

By (4.2), (4.9), and (4.17), we have

$$
\begin{aligned}
K_{0}-\inf (f) & \geq f\left(x_{0}\right)-f\left(x_{N_{0}}\right) \\
& =\sum_{i=0}^{N_{0}-1}\left(f\left(x_{i}\right)-f\left(x_{i+1}\right)\right) \\
& \geq \gamma \beta_{1} N_{0},
\end{aligned}
$$

and $N_{0} \leq\left(\beta_{1} \gamma\right)^{-1}\left(K_{0}-\inf (f)\right)$. This contradicts (4.7). The contradiction we have reached proves that there exists $j \in\left\{0, \ldots, N_{0}\right\}$ such that $f\left(x_{j}\right) \leq \inf (f)+\varepsilon / 4$. We show that, for all integers $i \in\{j, \ldots, n\}, f\left(x_{i}\right) \leq \inf (f)+\varepsilon$. Assume the contrary. Then there is an integer $p \in$ $\{j, \ldots, n\}$ for which

$$
f\left(x_{p}\right)>\inf (f)+\varepsilon .
$$

Hence, $p>j$. We may assume without loss of generality that $f\left(x_{i}\right) \leq \inf (f)+\varepsilon$ for all $i=$ $j, \ldots, p-1$, and, in particular,

$$
f\left(x_{p-1}\right) \leq \inf (f)+\varepsilon
$$

By (4.1) and (4.19), we have

$$
\left\|x_{p-1}\right\| \leq K-2
$$

There are two cases:

$$
f\left(x_{p-1}\right)>\inf (f)+\varepsilon / 4,
$$

and

$$
f\left(x_{p-1}\right) \leq \inf (f)+\varepsilon / 4 .
$$


Set $y=a_{p-1}^{-1}\left(x_{p}-x_{p-1}\right)$. It follows from (4.8), (4.10), and (4.11) that

$$
\begin{aligned}
\left\|y-V\left(x_{p-1}\right)\right\| & =\left\|a_{p-1}^{-1}\left(x_{p}-x_{p-1}\right)-V\left(x_{p-1}\right)\right\| \\
& \leq a_{p-1}^{-1}\left\|\left(x_{p}-x_{p-1}\right)-a_{p-1} V\left(x_{p-1}\right)\right\| \\
& \leq \beta_{1}^{-1} \delta \leq \delta_{0},
\end{aligned}
$$

and $x_{p}=x_{p-1}+a_{p-1} y$. Assume that (4.21) holds. In view of property (a), (4.4), (4.6), (4.10), (4.20), (4.21), and (4.23), we obtain that

$$
f\left(x_{p-1}\right)-f\left(x_{p}\right)=f\left(x_{p-1}\right)-f\left(x_{p-1}+a_{p-1} y\right) \geq a_{p-1} \gamma,
$$

which together with (4.19) implies that

$$
f\left(x_{p}\right) \leq f\left(x_{p-1}\right) \leq \inf (f)+\varepsilon .
$$

This contradicts (4.18). The contradiction we have reached proves that (4.22) is true. In view of (4.2) and (4.20), we have $\left\|V\left(x_{p-1}\right)\right\| \leq K_{0}$. By (4.10), ((4.20), and (4.23), we have

$$
\begin{aligned}
\left\|x_{p}\right\| & \leq\left\|x_{p-1}\right\|+a_{p-1}\|y\| \\
& \leq K-2+\beta_{0}\left(\left\|V\left(x_{p-1}\right)\right\|+\delta_{0}\right) \\
& \leq K-2+\beta_{0}\left(K_{0}+1\right) .
\end{aligned}
$$

It follows from (4.5), (4.6), (4.10), (4.11), (4.20), (4.22), (4.23), and (4.24) that

$$
\begin{aligned}
f\left(x_{p}\right) & \leq f\left(x_{p-1}\right)+\left|f\left(x_{p-1}\right)-f\left(x_{p}\right)\right| \\
& \leq \inf (f)+\varepsilon / 4+L_{0}\left\|x_{p-1}-x_{p}\right\| \\
& \leq \inf (f)+\varepsilon / 4+L_{0} a_{p-1}\|y\| \\
& \leq \inf (f)+\varepsilon / 4+L_{0} \beta_{0}\left(K_{0}+1\right) \\
& \leq \inf (f)+\varepsilon / 2 .
\end{aligned}
$$

This contradicts (4.18). The contradiction we have reached proves that $f\left(x_{i}\right) \leq \inf (f)+\varepsilon$ for all integers $i=j, \ldots, n$. This completes the proof of the theorem.

\section{THE SECOND RESULT}

Theorem 5.1. Assume that $f(x) \rightarrow \infty$ as $\|x\| \rightarrow \infty$, a vector field $V \in \mathscr{A}$ is regular, assumption A(ii) is valid and that at least one of the following conditions holds: $1 . V \in \mathscr{A}_{\text {au }} ; 2$. A(iii) is valid. Let $K, \varepsilon>0$ be given. Then there exists $\bar{\alpha} \in(0,1]$ such that the following assertion holds. Assume that a sequence $\left\{a_{i}\right\}_{i=0}^{\infty} \subset(0,1]$ satisfies

$$
\sum_{i=1}^{\infty} a_{i}=\infty, a_{i} \leq \bar{\alpha}, i=0,1, \ldots
$$

Then there exist $\delta>0$ and a natural number $N_{0}$ such that, for each sequence $\left\{x_{i}\right\}_{i=0}^{\infty} \subset X$, which satisfies $\left\|x_{0}\right\| \leq K$ and $\left\|x_{i+1}-x_{i}-a_{i} V\left(x_{i}\right)\right\| \leq a_{i} \delta$ for each integer $i \geq 0$, the inequality $f\left(x_{i}\right) \leq \inf (f)+\varepsilon$ holds for all integers $i \geq N_{0}$.

Proof. We may assume without loss of generality that $\varepsilon<1, K>2$, and

$$
\{x \in X: f(x) \leq \inf (f)+4\} \subset B(0, K-2) .
$$


Let

$$
K_{0}>\sup \{f(x): x \in B(0, K+1)\}+\sup \{\|V(x)\|: x \in B(0, K+1)\}+1 .
$$

Set

$$
E_{0}:=\left\{x \in X: f(x) \leq K_{0}+1\right\} .
$$

Clearly, the set $E_{0}$ is bounded and closed. Choose

$$
K_{1}>\max \left\{\sup \left\{\|x\|: x \in E_{0}\right\}+1+K, \sup \left\{\|V(x)\|: x \in E_{0}\right\}\right\} .
$$

There exists $L_{0} \geq 1$ such that

$$
\left\|f\left(z_{1}\right)-f\left(z_{2}\right) \mid \leq L_{0}\right\| z_{1}-z_{2} \| \text { for all } z_{1}, z_{2} \in B(0, K+2) .
$$

Lemma 3.1 implies that there exist positive numbers $\bar{\alpha}, \delta_{0} \in(0,1)$ and $\gamma>0$ such that the following property holds:

(a) for each point $x \in X$ satisfies $\|x\| \leq K_{1}, f(x) \geq \inf (f)+\varepsilon / 4$, each number $\alpha \in(0, \bar{\alpha}]$ and each point $y \in B\left(V(x), \delta_{0}\right), f(x)-f(x+\alpha y) \geq \alpha \gamma$.

We may assume that $\bar{\alpha} \leq\left(4 K_{0}+4\right)^{-1} L_{0}^{-1} \varepsilon$. Let $\left\{a_{i}\right\}_{i=0}^{\infty} \subset(0,1]$ satisfy (5.1). Hence, there exists a natural number $N_{0}>4$ such that

$$
\sum_{i=2}^{N_{0}-1} a_{i}>\gamma^{-1}\left(K_{0}-\inf (f)\right) .
$$

Choose a positive number $\delta \leq \delta_{0}$. Assume that $\left\{x_{i}\right\}_{i=0}^{\infty} \subset X$ satisfies $\left\|x_{0}\right\| \leq K$, and

$$
\left\|x_{i+1}-x_{i}-a_{i} V\left(x_{i}\right)\right\| \leq a_{i} \delta
$$

for each integer $i \geq 0$. We show that $f\left(x_{i}\right) \leq \inf (f)+\varepsilon$ for all integers $i \geq N_{0}$.

First we show that the following property holds:

(b) if

$$
i \geq 0 \text { is an integder, }\left\|x_{i}\right\| \leq K_{1}, f\left(x_{i}\right) \geq \inf (f)+\varepsilon / 4,
$$

then $f\left(x_{i}\right)-f\left(x_{i+1}\right) \geq a_{i} \gamma$. Assume that $i \geq 0$ be an integer, $\left\|x_{i}\right\| \leq K_{1}, f\left(x_{i}\right) \geq \inf (f)+\varepsilon / 4$. Set $y_{i}=a_{i}^{-1}\left(x_{i+1}-x_{i}\right)$. This implies from (5.8) that $x_{i+1}=x_{i}+a_{i} y_{i}$, and

$$
\begin{aligned}
\left\|y_{i}-V\left(x_{i}\right)\right\| & =\left\|a_{i}^{-1}\left(x_{i+1}-x_{i}\right)-V\left(x_{i}\right)\right\| \\
& \leq a_{i}^{-1}\left\|\left(x_{i+1}-x_{i}\right)-a_{i} V\left(x_{i}\right)\right\| \\
& \leq \delta
\end{aligned}
$$

It follows from property (a), (5.1), and (5.9) that

$$
f\left(x_{i}\right)-f\left(x_{i+1}\right)=f\left(x_{i}\right)-f\left(x_{i}+a_{i} y\right) \geq a_{i} \gamma .
$$

Thus property (b) holds. Now let us show that there exists an integer $i \in\left[0, N_{0}\right]$ such that $f\left(x_{i}\right) \leq \inf (f)+\varepsilon / 4$. Assume the contrary. Then

$$
f\left(x_{i}\right)>\inf (f)+\varepsilon / 4, i=0, \ldots, N_{0} .
$$

We show that $\left\|x_{i}\right\| \leq K_{1}, i=0, \ldots, N_{0}$. Assume the contrary. Then there exists an integer $p \in$ $\left[0, N_{0}\right]$ such that $\left\|x_{p}\right\|>K_{1}$. In view of (5.5), we have $p>0$. We may assume without loss of generality that $\left\|x_{i}\right\| \leq K_{1}, i=0, \ldots, p-1$. Property (b) and (5.10) imply that, for each $i \in$ $\{0, \ldots, p-1\}, f\left(x_{i+1}\right) \leq f\left(x_{i}\right)$, which together with (5.3) implies that $f\left(x_{p}\right) \leq f\left(x_{0}\right) \leq K_{0}$. From 
(5.4) and (5.5), we have $\left\|x_{p}\right\| \leq K_{1}$. This is a contradiction. Hence, $\left\|x_{i}\right\| \leq K_{1}, i=0, \ldots, N_{0}$. Property (b) and (5.10) imply that, for all $i=0, \ldots, N_{0}-1$,

$$
f\left(x_{i}\right)-f\left(x_{i+1}\right) \geq a_{i} \gamma .
$$

By (5.3) and (5.11), we have

$$
\begin{aligned}
K_{0}-\inf (f) & \geq f\left(x_{0}\right)-f\left(x_{N_{0}}\right) \\
& =\sum_{i=0}^{N_{0}-1}\left(f\left(x_{i}\right)-f\left(x_{i+1}\right)\right) \\
& \geq \gamma \sum_{i=0}^{N_{0}-1} a_{i}
\end{aligned}
$$

and

$$
\sum_{i=0}^{N_{0}-1} a_{i} \leq \gamma^{-1}\left(K_{0}-\inf (f)\right) .
$$

This contradicts (5.7). Hence, there exists $j \in\left\{0, \ldots, N_{0}\right\}$ such that $f\left(x_{j}\right) \leq \inf (f)+\varepsilon / 4$. We show that, for all integers $i \geq j, f\left(x_{i}\right) \leq \inf (f)+\varepsilon$. Assume the contrary. Then there is an integer $p \geq j$ for which $f\left(x_{p}\right)>\inf (f)+\varepsilon$. It follows that $p>j$. We may assume without loss of generality that

$$
f\left(x_{i}\right) \leq \inf (f)+\varepsilon \text { for all } i=j, \ldots, p-1 .
$$

By (5.2) and (5.12), we have, for all $i=j, \ldots, p-1,\left\|x_{i}\right\| \leq K-2$. There are two cases:

$$
f\left(x_{p-1}\right) \geq \inf (f)+\varepsilon / 4
$$

and

$$
f\left(x_{p-1}\right)<\inf (f)+\varepsilon / 4 \text {. }
$$

If (5.13) holds, we obtain from property (b), and (5.12) that $f\left(x_{p}\right) \leq f\left(x_{p-1}\right) \leq \inf (f)+\varepsilon$. This is a contradiction. Hence, (5.14) is true. In view of (5.1), (5.3), and (5.10), we have

$$
\left\|x_{p}-x_{p-1}\right\| \leq a_{p-1}\left\|V\left(x_{p-1}\right)+1\right\| \leq a_{p-1}\left(K_{0}+1\right),
$$

and $\left\|x_{p}\right\| \leq K-1$. In view of (5.1) and (5.6), we have

$$
\begin{aligned}
f\left(x_{p}\right) & \leq f\left(x_{p-1}\right)+\left|f\left(x_{p-1}\right)-f\left(x_{p}\right)\right| \\
& \leq \inf (f)+\varepsilon / 4+L_{0}\left\|x_{p-1}-x_{p}\right\| \\
& \leq \inf (f)+\varepsilon / 4+(\varepsilon / 4) L_{0}\left(K_{0}+1\right) \bar{\alpha} \\
& \leq \inf (f)+\varepsilon / 2 .
\end{aligned}
$$

This is a contradiction. Hence, $f\left(x_{i}\right) \leq \inf (f)+\varepsilon$ for all integers $i \geq j$. This completes the proof.

Remark 5.1. In our main results, $\delta$ depends on $\varepsilon$, but we cannot obtain an explicit dependence for them. This is natural because $\delta$ also depends on the regular vector field $V$. Moreover, in the definition of a regular vector field, we do not have explicit dependence $\delta(n)$ on $n$. Note that, for gradient-like vector fields, such dependence between $\delta$ and $\varepsilon$ exists and was studied in [13, 14]. 


\section{REFERENCES}

[1] S. Reich, A.J. Zaslavski, Generic convergence of descent methods in Banach spaces, Math. Oper. Res. 25 (2000), 231-242.

[2] S. Reich, A.J. Zaslavski, The set of divergent descent methods in a Banach space is $\sigma$-porous, SIAM J. Optim. 11 (2001), 1003-1018.

[3] B. S. Mordukhovich, Variational Analysis and Generalized Differentiation. I: Basic Theory, Springer, Berlin, 2006.

[4] M. Bačák, A variational approach to stochastic minimization of convex functionals, Pure Appl. Funct. Anal. 3 (2018), 287-295.

[5] A. Cegielski, A method of projection onto an acute cone with level control in convex minimization, Math. Program. 85 (1999), 469-490.

[6] H.B. Curry, The method of steepest descent for nonlinear minimization problems, Quarterly Appl. Math. 2 (1944), 258-261.

[7] A. Gibali, K.-H. Kufer, D. Reem, P. Suss, A generalized projection-based scheme for solving convex constrained optimization problems, Comput. Optim. Appl. 70 (2018), 737-762.

[8] S.-M. Grad, E.-L. Pop, Vector duality for convex vector optimization problems by means of the quasi-interior of the ordering cone, Optimization 63 (2014), 21-37.

[9] I. Griva, Convergence analysis of augmented Lagrangian - fast projected gradient method for convex quadratic problems, Pure Appl. Funct. Anal. 3 (2018), 417-428.

[10] Y. Hu, C. Li, X. Yang, On convergence rates of linearized proximal algorithms for convex composite optimization with applications, SIAM J. Optim. 26 (2016), 1207-1235.

[11] I. Konnov, On convergence properties of a subgradient method, Optim. Meth. Softw. 18 (2003), 53-62.

[12] R. Polyak, Exterior distance function, Pure Appl. Funct. Anal. 2 (2017), 369-394.

[13] A.J. Zaslavski, Numerical Optimization with Computational Errors, Springer, 2016.

[14] A.J. Zaslavski, Convex Optimization with Computational Errors, Springer Optimization and Its Applications, Springer, 2020.

[15] C. Zalinescu, On Lagrange multipliers in convex entropy minimization, Pure Appl. Funct. Anal. 3 (2018), 393-402.

[16] J.-B. Hiriart-Urruty, C. Lemaréchal, Convex Analysis and Minimization Algorithms, Springer, Berlin, 1993.

[17] S. Reich, A.J. Zaslavski, Genericity in Nonlinear Analysis, Developments in Mathematics, 34, Springer, New York, 2014.

[18] A.J. Zaslavski, Optimization on Metric and Normed Spaces, Springer, New York, 2010.

[19] S. Reich, A.J. Zaslavski, Regular vector-fields in Banach spaces, Taiwanese J. Math. 12 (2008), 1165-1176.

[20] S. Reich, A.J. Zaslavski, Descent methods with computational errors in Banach spaces, Optimization 14 (2019), 41-50. 